\title{
Duplication coefficients via generating functions
}

\author{
HAMZA CHAGGARA $\dagger$ and WOLFRAM KOEPF* \\ †Département de Math-Physique, Institut Préparatoire aux Études \\ d'Ingénieur de Monastir, 5019 Monastir, Tunisia \\ †Fachbereich Mathematik, Universität Kassel, D-34109 Kassel, Germany \\ Communicated by R. P. Gilbert
}

(Received in final form 15 December 2006)

In this article, we solve the duplication problem,

$$
P_{n}(a x)=\sum_{m=0}^{n} C_{m}(n, a) P_{m}(x),
$$

where $\left\{P_{n}\right\}_{n>0}$ belongs to a wide class of polynomials, including the classical orthogonal polynomials (Hermite, Laguerre, Jacobi) as well as the classical discrete orthogonal polynomials (Charlier, Meixner, Krawtchouk) for the specific case $a=-1$. We give closed-form expressions as well as recurrence relations satisfied by the duplication coefficients.

Keywords: Duplication coefficients; Generating functions; Brenke polynomials; Jacobi polynomials; Charlier polynomials; Meixner polynomials; Krawtchouk polynomials

MSC: $33 \mathrm{C} 45 ; 33 \mathrm{C} 20 ; 05 \mathrm{~A} 15$

\section{Introduction}

Let $\mathscr{P}$ be the linear space of polynomials with complex coefficients. A polynomial sequence $\left\{P_{n}\right\}_{n \geq 0}$ in $\mathscr{P}$ is called a polynomial set if and only if $\operatorname{deg} P_{n}=n$ for all nonnegative integers $n$.

Given a polynomial set $\left\{P_{n}\right\}_{n \geq 0}$, the so-called duplication or multiplication problem associated with this family asks to find the coefficients $C_{m}(n, a)$ in the expansion

$$
P_{n}(a x)=\sum_{m=0}^{n} C_{m}(n, a) P_{m}(x),
$$

where $a$ designates a nonzero complex number.

*Corresponding author. Email: koepf@mathematik.uni-kassel.de 
Such identities have applications in many problems in pure and applied mathematics, especially in combinatorial analysis. This problem may be viewed as a special case of the so-called connection problem between two polynomial sets where the first member of (1.1) is replaced by a polynomial set $Q_{n}(x)$.

The solution of this problem is known for some particular polynomial sets. For instance, the so-called Fields-Wimp expansion [10] gives a solution for some hypergeometric polynomials [17].

A general method, based on lowering operators and generating functions, was developed in [2,3] to solve connection and linearization problems [4,7]. The purpose of this work is to use this approach to express explicitly the coefficients $C_{m}(n, a)$. The method depends on simple manipulations of formal power series. The approach we shall propose in this article does not need the orthogonality of the polynomials involved in the problem, and in this way the formulae obtained are still valid outside the range of orthogonality of the parameters.

\section{The method}

Definition 2.1 Let $\left\{P_{n}\right\}_{n \geq 0}$ be a polynomial set. $\left\{P_{n}\right\}_{n \geq 0}$ is said to have a generating function of Boas-Buck type (or is called a Boas-Buck polynomial set) if there exists a sequence of nonzero numbers $\left(\lambda_{n}\right)_{n \geq 0}$ such that

$$
\sum_{n=0}^{\infty} \lambda_{n} P_{n}(x) t^{n}=A(t) B(x C(t)),
$$

where $A, B, C$ are three formal power series such that

$$
A(0) C^{\prime}(0) \neq 0, C(0)=0 \quad \text { and } \quad B^{(k)}(0) \neq 0, k \in \mathbb{N}
$$

The choice of $C(t)=t$ gives the class of Brenke polynomials.

It is obvious to see that if the normalization is changed, say: $P_{n}=c_{n} \widetilde{P}_{n}$, then the new duplication coefficients $\widetilde{C}_{m}(n, a)$ are given by

$$
\widetilde{C}_{m}(n, a)=\frac{c_{m}}{c_{n}} C_{m}(n, a)
$$

It means that there is no loss of generality if we limit ourselves to the case $\lambda_{n}=1 / n$ ! in (2.1).

Theorem 2.2 Let $\left\{P_{n}\right\}_{n \geq 0}$ be a Boas-Buck polynomial set generated by (2.1). Then the associated duplication coefficients defined by (1.1) are given by

$$
\mathcal{F}(t)=\frac{A(t)}{A(\Phi(t))} \Phi^{m}(t)=\sum_{n=m}^{\infty} \frac{m !}{n !} C_{m}(n, a) t^{n}
$$

where $\Phi(t)=C^{-1}(a C(t))$ and $C^{-1}$ is the inverse of $C$, i.e., $C^{-1}(C(t))=C\left(C^{-1}(t)\right)=t$. 
Proof The proof of this result is based on the following lemma.

Lemma 2.3 (see [3], Corollary 3.9) Let $\left\{P_{n}\right\}_{n \geq 0}$ and $\left\{Q_{n}\right\}_{n \geq 0}$ be two polynomial sets of Boas-Buck type that are generated, respectively, by

$$
A_{1}(t) B\left(x C_{1}(t)\right)=\sum_{n=0}^{\infty} \frac{P_{n}(x)}{n !} t^{n} \quad \text { and } \quad A_{2}(t) B\left(x C_{2}(t)\right)=\sum_{n=0}^{\infty} \frac{Q_{n}(x)}{n !} t^{n}
$$

Then the connection coefficients in

$$
Q_{n}(x)=\sum_{m=0}^{n} C_{m}(n) P_{m}(x)
$$

are given by

$$
\frac{A_{2}(t)}{A_{1}(\Phi(t))} \Phi^{m}(t)=\sum_{k=m}^{\infty} \frac{m !}{k !} C_{m}(k) t^{k}
$$

where $\Phi(t)=C_{1}^{-1}\left(C_{2}(t)\right)$.

In order to derive (2.3) from this lemma, we set $A_{1}=A_{2}=A, C_{1}=C$ and $C_{2}=a C$ in (2.5).

This result shows that the duplication coefficients of a Boas-Buck polynomial set generated by (2.1) depend only on $C$ and $A$.

\section{Applications}

\subsection{Brenke polynomials}

COROLlary 3.1 The Brenke polynomials $\left\{P_{n}\right\}_{n \geq 0}$, generated by

$$
A(t) B(x t)=\sum_{n=0}^{\infty} \frac{P_{n}(x)}{n !} t^{n}
$$

possess a duplication formula of the form

$$
P_{n}(a x)=\sum_{m=0}^{n}\left(\begin{array}{l}
n \\
m
\end{array}\right) a^{m} \beta_{n-m}(a) P_{m}(x)
$$

where

$$
\frac{A(t)}{A(a t)}=\sum_{k=0}^{\infty} \frac{\beta_{k}(a)}{k !} t^{k}
$$


Proof Applying Theorem 2.2 with $C(t)=t$, we obtain $\Phi(t)=a t$ and

$$
\frac{A(t)}{A(a t)} a^{m} t^{m}=\sum_{n=m}^{\infty} \frac{m !}{n !} C_{m}(n, a) t^{n}
$$

Put $A(t) / A(a t)=\sum_{k=0}^{\infty}\left(\beta_{k}(a) / k !\right) t^{k}$. It follows, by identification, that $C_{m}(n, a)=$ $\left(\begin{array}{c}n \\ m\end{array}\right) a^{m} \beta_{n-m}$.

For $A(t)=e^{t}$, Corollary 3.1 is reduced to Carlitz formula [6]

$$
P_{n}(a x)=\sum_{m=0}^{n}\left(\begin{array}{l}
n \\
m
\end{array}\right) a^{m}(1-a)^{n-m} P_{m}(x) .
$$

Let us mention that Corollary 3.1 was already given in [3] and applied essentially to some $q$-polynomial sets.

Next, we consider some examples.

3.1.1. Brafman polynomials. The Brafman polynomials defined by

$$
\mathscr{B}_{n}^{1}\left(\left(a_{p}\right),\left(b_{q}\right) ; x\right)={ }_{p+1} F_{q}\left(\begin{array}{c}
-n,\left(a_{p}\right) \\
\left(b_{q}\right)
\end{array} \mid x\right)
$$

are generated by $[5,8]$

$$
\sum_{n=0}^{\infty} \mathscr{B}_{n}^{1}\left(\left(a_{p}\right),\left(b_{q}\right) ; x\right) \frac{t^{n}}{n !}=e_{p}^{t} F_{q}\left(\begin{array}{c}
\left(a_{p}\right) \\
\left(b_{q}\right)
\end{array} \mid-x t\right)
$$

For the definition of the generalized hypergeometric function ${ }_{p} F_{q}$ see [15] or [18].

In the given case, we have $A(t)=e^{t}$. According to (3.3), we obtain

$$
\mathscr{B}_{n}^{1}\left(\left(a_{p}\right),\left(b_{q}\right) ; a x\right)=\sum_{m=0}^{n}\left(\begin{array}{l}
n \\
m
\end{array}\right) a^{m}(1-a)^{n-m} \mathscr{B}_{m}^{1}\left(\left(a_{p}\right),\left(b_{q}\right) ; x\right) .
$$

A particular case of the Brafman polynomials are the Laguerre polynomials generated by $([18$, p. 201])

$$
e_{0}^{t} F_{1}\left(\begin{array}{c}
- \\
\alpha+1
\end{array} \mid-x t\right)=\sum_{n=0}^{\infty} \frac{L_{n}^{(\alpha)}(x)}{(\alpha+1)_{n}} t^{n}
$$

According to (3.3) or (3.6), we find the well-known formula ([18, p. 209])

$$
L_{n}^{(\alpha)}(a x)=\sum_{m=0}^{n} \frac{(\alpha+1)_{n}}{(n-m) !(\alpha+1)_{m}} a^{m}(1-a)^{n-m} L_{m}^{(\alpha)}(x) .
$$


3.1.2. Chaunday polynomials. The Chaunday hypergeometric polynomials

$$
P_{n}^{\lambda}(x)={ }_{p+1} F_{q+1}\left(\begin{array}{c}
-n,\left(a_{p}\right) \\
1-\lambda-n,\left(b_{q}\right)
\end{array} \mid x\right)
$$

are generated by [8]

$$
\sum_{n=0}^{\infty} \frac{(\lambda)_{n}}{n !} P_{n}^{\lambda}(x) t^{n}=(1-t)_{p}^{-\lambda} F_{q}\left(\begin{array}{c}
\left(a_{p}\right) \\
\left(b_{q}\right)
\end{array} \mid x t\right)
$$

For this case, we have $A(t)=(1-t)^{-\lambda}$ and

$$
\frac{A(t)}{A(a t)}=\left(\frac{1-t}{1-a t}\right)^{-\lambda}
$$

It follows, using a Cauchy product,

$$
\begin{aligned}
(1-t)^{-\lambda}(1-a t)^{\lambda} & =\sum_{n=0}^{\infty} \frac{(\lambda)_{n}}{n !} t^{n} \sum_{n=0}^{\infty} \frac{(-\lambda)_{n}}{n !} a^{n} t^{n}=\sum_{n=0}^{\infty}\left(\sum_{k=0}^{n} \frac{(\lambda)_{n-k}}{(n-k) !} \frac{(-\lambda)_{k}}{k !} a^{k}\right) t^{n} \\
& =\sum_{n=0}^{\infty} \frac{(\lambda)_{n}}{n !}\left(\sum_{k=0}^{n} \frac{(-n)_{k}(-\lambda)_{k}}{(1-\lambda-n)_{k}} \frac{a^{k}}{k !}\right) t^{n}=\sum_{n=0}^{\infty} \frac{(\lambda)_{n}}{n !}{ }_{2} F_{1}\left(\begin{array}{c}
-n,-\lambda \\
1-\lambda-n
\end{array} \mid a\right) t^{n} .
\end{aligned}
$$

Note that this type of computation can be done completely automatically by the Sumtohyper command of the Maple hsum package [15]. We obtain from (3.1)

$$
(\lambda)_{n} P_{n}^{\lambda}(a x)=\sum_{m=0}^{n}\left(\begin{array}{l}
n \\
m
\end{array}\right) a^{m}(\lambda)_{n-m}{ }_{2} F_{1}\left(\begin{array}{c}
m-n,-\lambda \\
1-\lambda-n+m
\end{array} \mid a\right)(\lambda)_{m} P_{m}^{\lambda}(x) .
$$

It is easy to obtain the recurrence equation

$$
a m(m+1) \beta_{m}(a)-(\lambda+a m+a+m+1-\lambda a) \beta_{m+1}(a)+\beta_{m+2}(a)=0
$$

for the coefficients $\beta_{k}(a)$ defined by (3.2) in the given situation. This can be accomplished at least in two ways: either we use the given generating function $F(t):=$ $A(t) / A(a t)$ of $\beta_{k}(a) / k$ !, compute the holonomic differential equation (i.e., linear, homogeneous with polynomial coefficients)

$$
(-1+t)(-1+a t) F^{\prime}(t)+\lambda(a-1) F(t)=0
$$

for $F(t)$ and convert this differential equation into the above holonomic recurrence equation for the corresponding series coefficients by the Maple FPS package [12,14]. 
Or we use the hypergeometric representation of $\beta_{k}(a)$ given by (3.8) and (3.2) together with Zeilberger's algorithm (see e.g., [15]) via the Maple sumrecursion command which yields the same recurrence.

3.1.3. Gould-Hopper polynomials. Recall that the Gould-Hopper polynomials are generated by [11]

$$
e^{h t^{d}} \exp (x t)=\sum_{n=0}^{\infty} g_{n}^{d}(x, h) \frac{t^{n}}{n !}, \quad d \in \mathbb{N} .
$$

For this case we have $A(t)=e^{h t^{d}}$ and

$$
\frac{A(t)}{A(a t)}=e^{h\left(1-a^{d}\right) t^{d}}=\sum_{k=0}^{\infty} \frac{h^{k}\left(1-a^{d}\right)^{k}}{k !} t^{k d}
$$

which, by virtue of (3.1), gives

$$
g_{n}^{d}(a x, h)=\sum_{m=0}^{[n / d]} \frac{a^{n} n !}{m !(n-d m) !} h^{m}\left(a^{-d}-1\right)^{m} g_{n-d m}^{d}(x, h) .
$$

This family contains as a special case the Hermite polynomials $H_{n}(x)=g_{n}^{2}(2 x,-1)$, so (3.12) is reduced to

$$
H_{n}(a x)=\sum_{m=0}^{[n / 2]} \frac{a^{n} n !}{(n-2 m) ! m !}\left(1-a^{-2}\right)^{m} H_{n-2 m}(x) .
$$

\subsection{Shifted Jacobi polynomials}

The shifted Jacobi polynomials defined by [18]

$$
R_{n}^{(\alpha, \beta)}(x)=P_{n}^{(\alpha, \beta)}(1-x)=\frac{(\alpha+1)_{n}}{n !}{ }_{2} F_{1}\left(\begin{array}{c|c}
-n, \alpha+\beta+n+1 \\
\alpha+1
\end{array}\right)
$$

are generated by

$$
(1-t)_{2}^{-\lambda} F_{1}\left(\begin{array}{c|c}
\frac{\lambda}{2}, \frac{\lambda+1}{2} & \frac{-2 x t}{(1-t)^{2}}
\end{array}\right)=\sum_{n=0}^{\infty} \frac{(\lambda)_{n} R_{n}^{(\alpha, \beta)}(x)}{(1+\alpha)_{n}} t^{n},
$$

where $\lambda=\alpha+\beta+1$.

To solve the duplication problem for the shifted Jacobi case, we need the following lemma.

LEMMA 3.2 (Lagrange's inversion formula [20]) Let $\xi$ be a function of $t$ implicitly defined by

$$
\xi=t(1+\xi)^{s+1}, \quad \xi(0)=0
$$


Then, we have

$$
(1+\xi(t))^{r}=\sum_{n=0}^{\infty} \frac{r}{r+(s+1) n}\left(\begin{array}{c}
r+(s+1) n \\
n
\end{array}\right) t^{n},
$$

where $r$ and s are complex numbers independent of $n$.

For this case we have

$$
A(t)=(1-t)^{-\lambda} \quad \text { and } \quad C(t)=\frac{-t}{(1-t)^{2}}
$$

$C^{-1}$ is implicitly defined by

$$
\left(1-C^{-1}(t)\right)^{2} t=-C^{-1}(t)
$$

Using (3.14), with $\xi=-C^{-1}, s=1$ and $r=\lambda+2 m$, we obtain

$$
\begin{aligned}
\frac{\left[C^{-1}\right]^{m}(t)}{A\left(C^{-1}(t)\right)} & =(-1)^{m}\left(1-C^{-1}(t)\right)^{2 m+\lambda} t^{m} \\
& =(-1)^{m} \sum_{n=0}^{\infty} \frac{\lambda+2 m}{\lambda+2 n+2 m}\left(\begin{array}{c}
2 n+2 m+\lambda \\
n
\end{array}\right) t^{n+m}
\end{aligned}
$$

Replacing $t$ by $a C(t)$ and multiplying by $A(t)$, we get moreover

$$
\begin{aligned}
\mathcal{F}(t) & =(1-t)^{-\lambda}(-1)^{m} \sum_{n=0}^{\infty} \frac{\lambda+2 m}{\lambda+2 n+2 m}\left(\begin{array}{c}
2 n+2 m+\lambda \\
n
\end{array}\right)(a C(t))^{n+m} \\
& =\sum_{n=0}^{\infty} \frac{\lambda+2 m}{\lambda+2 n+2 m}\left(\begin{array}{c}
2 n+2 m+\lambda \\
n
\end{array}\right) a^{n+m}(-1)^{n} \frac{t^{n+m}}{(1-t)^{2 n+2 m+\lambda}} \\
& =\sum_{n=0}^{\infty} \sum_{k=0}^{\infty} \frac{(2 n+2 m+\lambda)_{k}}{\lambda+2 n+2 m}\left(\begin{array}{c}
2 n+2 m+\lambda \\
n
\end{array}\right) a^{n+m}(-1)^{n} t^{n+m+k} \\
& =\sum_{n=0}^{\infty} \sum_{k=0}^{n} \frac{(2 k+2 m+\lambda)_{n-k}}{\lambda+2 m+2 m} \frac{\lambda+2 k}{(n-k) !}\left(\begin{array}{c}
2 m+2 k+\lambda \\
k
\end{array}\right) a^{m+k}(-1)^{k} t^{n+m} \\
& =\sum_{n=m}^{\infty}\left(\sum_{k=0}^{n-m} \frac{(2 m+2 k+\lambda)_{n-m-k}}{\lambda+2 m+2 k}\left(\begin{array}{c}
2 m+2 k+\lambda \\
k
\end{array}\right) a^{m+k}(-1)^{k}\right) t^{n} .
\end{aligned}
$$


The duplication formula associated with shifted Jacobi polynomials can therefore be written in terms of hypergeometric functions as follows

$$
R_{n}^{(\alpha, \beta)}(a x)=\sum_{m=0}^{n} \frac{a^{m}}{(n-m) !} \frac{(1+\alpha)_{n}}{(1+\alpha)_{m}} \frac{(\lambda+n)_{m}}{(\lambda+m)_{m}}{ }_{2} F_{1}\left(\begin{array}{c}
m-n, m+n+\lambda \\
2 m+\lambda+1
\end{array} \mid a\right) R_{m}^{(\alpha, \beta)}(x)
$$

For $C_{m}(n, a)$ we get (again by Zeilberger's algorithm) the recurrence

$$
\begin{aligned}
& a(m-n)(\alpha+\beta+5+2 m)(\alpha+\beta+2+m)(\alpha+\beta+1+m) \\
& \quad(2 m+4+\alpha+\beta)(m+n+\alpha+\beta+1) C_{m}(n)-(1+\alpha+m) \\
& \quad(\alpha+\beta+5+2 m)(\alpha+\beta+1+2 m)(\alpha+\beta+2+m) \\
& \quad \times\left(2 a m^{2}-4 m^{2}-4 m \beta+2 a m \alpha-12 m+2 a m \beta+6 a m-4 m \alpha-8+2 a \alpha n\right. \\
& \quad+2 a n+2 a n^{2}+2 a \beta n+4 a \beta+4 a \alpha+2 a \beta \alpha+4 a-6 \alpha \\
& \left.\quad-6 \beta+a \alpha^{2}+a \beta^{2}-\alpha^{2}-2 \beta \alpha-\beta^{2}\right) C_{m+1}(n)+a(2+m-n) \\
& \quad(2+\alpha+m)(1+\alpha+m)(2 m+\alpha+\beta+2)(\alpha+\beta+1+2 m) \\
& \quad(m+\alpha+3+\beta+n) C_{m+2}(n)=0
\end{aligned}
$$

w.r.t the variable $m$. The initial values for this recurrence are given by $C_{n}(n)=a^{n}$ and $C_{n+1}(n)=0$. In a similar way, the recurrence

$$
\begin{aligned}
& (2+\alpha+n)(1+\alpha+n)(4+2 n+\beta+\alpha)(m-n) \\
& (m+n+\alpha+\beta+1) C_{m}(n)-(2+\alpha+n)(\alpha+3+2 n+\beta) \\
& (\alpha+\beta+1+n)\left(a \alpha^{2}-\alpha^{2}+2 a \beta \alpha-2 \beta \alpha-2 \alpha n-4 \alpha-2 m \alpha\right. \\
& \quad+6 a \alpha+4 a \alpha n-4-2 m^{2}-2 m \beta-2 n^{2}-2 \beta n+12 a n \\
& \left.\quad+4 a n^{2}+4 a \beta n+6 a \beta+8 a-2 m-4 \beta-6 n+a \beta^{2}-\beta^{2}\right) \\
& \quad C_{m}(n+1)+(2 n+\alpha+\beta+2)(\alpha+\beta+2+n)(\alpha+\beta+1+n) \\
& (m-n-2)(m+\alpha+3+\beta+n) C_{m}(n+2)=0
\end{aligned}
$$

w.r.t $n$ is obtained, where we have replaced $\lambda$ by $\alpha+\beta+1$, again.

\subsection{Classical discrete orthogonal polynomials}

In this section, we limit ourselves to the following particular duplication problem

$$
P_{n}(-x)=\sum_{k=0}^{n} C_{k}(n) P_{k}(x)
$$

3.3.1. Charlier polynomials. The monic Charlier polynomial set $[9$, Chapter VI, (1.2)]

$$
\widetilde{C}_{n}^{(\alpha)}(x)=\sum_{m=0}^{n}\left(\begin{array}{l}
n \\
m
\end{array}\right)(-\alpha)^{n-m} m !\left(\begin{array}{l}
x \\
m
\end{array}\right) \quad(n \geq 0)
$$


is generated by [9, Chapter VI, (1.2)]

$$
G(x, t)=e^{-\alpha t} e^{x \ln (1+t)}=\sum_{n=0}^{\infty} \frac{\widetilde{C}_{n}^{(\alpha)}(x)}{n !} t^{n} .
$$

For this case, we have:

$$
A(t)=e^{-\alpha t}, C(t)=\ln (1+t), C^{-1}(t)=e^{t}-1 \quad \text { and } \quad \Phi(t)=-\frac{t}{t+1} .
$$

It follows that

$$
\mathcal{F}(t)=\frac{A(t)}{A(\Phi(t))} \Phi^{m}(t)=(1+t)^{-m} \exp \left(-\alpha \frac{t}{t+1}\right) e^{-\alpha t}(-t)^{m}
$$

By sum manipulations, we get

$$
\begin{aligned}
(1+t)^{-m} \exp \left(-\alpha \frac{t}{t+1}\right) & =\sum_{n=0}^{\infty} \frac{(-\alpha)^{n}}{n !} \frac{t^{n}}{(1+t)^{n+m}} \\
& =\sum_{n=0}^{\infty} \frac{(-\alpha)^{n}}{n !} t^{n}\left(\sum_{k=0}^{\infty}(-1)^{k} \frac{(n+m)_{k}}{k !} t^{k}\right) \\
& =\sum_{n=0}^{\infty}\left(\sum_{k=0}^{n}(-1)^{n} \frac{(m)_{n}}{(m)_{k}} \frac{\alpha^{k}}{k !(n-k) !}\right) t^{n}
\end{aligned}
$$

It follows

$$
\begin{aligned}
\mathcal{F}(t) & =\sum_{n=0}^{\infty}\left(\sum_{k=0}^{n}(-1)^{n} \frac{(m)_{n}}{(m)_{k}} \frac{\alpha^{k}}{k !(n-k) !}\right) t^{n} \sum_{n=0}^{\infty} \frac{(-\alpha)^{n}}{n !} t^{n}(-t)^{m} \\
& =\sum_{n=m}^{\infty}(-1)^{n}\left(\sum_{p=0}^{n-m} \sum_{k=0}^{p} \frac{(m)_{p}}{(m)_{k}} \frac{\alpha^{n-m+k-p}}{k !(p-k) !(n-m-p) !}\right) t^{n}
\end{aligned}
$$

Then the duplication coefficient in (3.16) is given by

$$
C_{m}(n)=(-1)^{n} \alpha^{n-m}\left(\begin{array}{c}
n \\
m
\end{array}\right) \sum_{k=0}^{n-m} \frac{(-n+m)_{k}(m)_{k}}{k !}{ }_{1} F_{1}\left(\begin{array}{c}
-k \\
m
\end{array} \mid-\alpha\right)(-\alpha)^{-k}
$$

Recently, a fourth order recurrence relation to calculate the connection coefficients in this last duplication formula was given in [1], where the authors used a different approach based on the so-called Navima algorithm. Unfortunately their recurrence [1, p. 386] contains a misprint and is wrong.

Using the above double sum and a Fasenmyer type algorithm [15] to deduce recurrence equations for multiple hypergeometric series [19,22] we get - using 
Sprenger's multsum package - the following much simpler third order recurrence for $C_{m}(n)$ :

$$
\begin{aligned}
& -\alpha(m+2)(m+1)(m+3) C_{m+3}(n) \\
& \quad-(m+2)(m+1)(1+m+2 \alpha) C_{m+2}(n) \\
& \quad+(m+1)(-1-2 m-2 \alpha+n) C_{m+1}(n)+(n-m) C_{m}(n)=0
\end{aligned}
$$

w.r.t $m$ with initial values $C_{n}(n)=(-1)^{n}$ and $C_{n+1}(n)=C_{n+2}(n)=0$, as well as*

$$
\begin{aligned}
& (n+3-m) C_{m}(n+3)+(n+2)(n+3)(n+1+2 \alpha) C_{m}(n+1) \\
& \quad+(n+2) \alpha(n+1)(n+3) C_{m}(n)+(n+3)(2 n+4+2 \alpha-m) C_{m}(n+2) \\
& \quad=0
\end{aligned}
$$

w.r.t $n$.

3.3.2. Meixner polynomials. The monic Meixner polynomial set [16, Theorem 6]

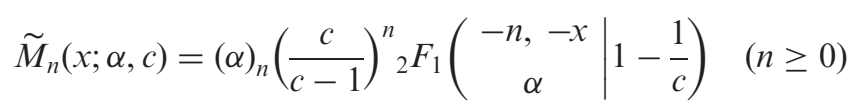

is generated by $[13,(1.9 .11)]$ :

$$
G(x, t)=\sum_{n=0}^{\infty}\left(\frac{c-1}{c}\right)^{n} \frac{\tilde{M}_{n}(x ; \alpha, c)}{n !} t^{n}=\frac{1}{(1-t)^{\alpha}} \exp \left(x \ln \frac{1-(t / c)}{1-t}\right) .
$$

For this case, we have

$$
A(t)=\frac{1}{(1-t)^{\alpha}}, C(t)=\ln \frac{1-(t / c)}{1-t}, C^{-1}(t)=\frac{c\left(e^{t}-1\right)}{e^{t} c-1} \quad \text { and } \quad \Phi(t)=\frac{c t}{-c+c t+t}
$$

It follows that

$$
\mathcal{F}(t)=\frac{A(t)}{A(\Phi(t))} \Phi^{m}(t)=\left(\frac{1-(t / c)}{1-t}\right)^{\alpha} \frac{(-t)^{m}}{(1-(1+(1 / c)) t)^{m+\alpha}}
$$

By sum manipulations (as in (3.8)), we get

$$
\mathcal{F}(t)=(-t)^{m} \sum_{n=0}^{\infty} \frac{(\alpha)_{n}}{n !}{ }_{2} F_{1}\left(\begin{array}{c|c}
-n,-\alpha & 1 \\
1-\alpha-n & \frac{c}{c}
\end{array}\right) t^{n} \sum_{n=0}^{\infty} \frac{(m+\alpha)_{n}}{n !}\left(1+\frac{1}{c}\right)^{n} t^{n}
$$

*Note that Maple sorts expressions by their memory allocation, therefore the output is not always in the usual order. 
which gives

$$
\mathcal{F}(t)=(-t)^{m} \sum_{n=0}^{\infty}\left(\sum_{k=0}^{n} \frac{(\alpha)_{k}}{k !}{ }_{2} F_{1}\left(\begin{array}{c|c}
-k,-\alpha \\
1-\alpha-k & \frac{1}{c}
\end{array}\right) \frac{(m+\alpha)_{n-k}}{(n-k) !}\left(1+\frac{1}{c}\right)^{n-k}\right) t^{n} .
$$

Then the duplication coefficient in (3.16) is given by

$$
C_{m}(n)=(-1)^{m} \frac{n !}{m !}\left(\frac{c+1}{c-1}\right)^{n-m} \sum_{k=0}^{n-m} \frac{(\alpha)_{k}(m+\alpha)_{n-m-k}}{k !(n-m-k) !}\left(1+\frac{1}{c}\right)^{-k}{ }_{2} F_{1}\left(\begin{array}{c|c}
-k,-\alpha \\
1-\alpha-k
\end{array} \mid \frac{1}{c}\right) .
$$

Note that in [1, p. 385] a similar representation was obtained.

Using the multsum package, we get the recurrence

$$
\begin{aligned}
& -(c-1)^{3}(n-m) C_{m}(n) \\
& -(m+2)(m+1)(c-1)\left(c n-c^{2} m-c^{2}-2 \alpha c^{2}-3 c m-4 c-2 c \alpha-1-m\right) C_{m+2}(n) \\
& -c(m+3)(m+2)(m+1)(m+2+\alpha)(c+1) C_{m+3}(n) \\
& \quad+(c-1)^{2}(m+1)(c n+n-2 c m-c-2 c \alpha-2 m-1) C_{m+1}(n)=0
\end{aligned}
$$

w.r.t $m$ with initial values $C_{n}(n)=(-1)^{n}$ and $C_{n+1}(n)=C_{n+2}(n)=0$, as well as

$$
\begin{aligned}
& c(n+2)(n+1)(n+3)(\alpha+n)(c+1) C_{m}(n) \\
& \quad-(n+3)(n+2)(c-1)\left(n c^{2}+c^{2}+3 c n+3 c+n+1+2 c^{2} \alpha+2 c \alpha-c m\right) C_{m}(n+1) \\
& \quad+(c-1)^{2}(n+3)(2 c n+4 c+2 n+4-c m+2 c \alpha-m) C_{m}(n+2) \\
& \quad-(c-1)^{3}(n+3-m) C(n+3)=0
\end{aligned}
$$

w.r.t $n$.

3.3.3. Krawtchouk polynomials. The monic Krawtchouk polynomial set [16, Theorem 6]

$$
\widetilde{K}_{n}^{p}(x, N)=p^{n}(-N)_{n 2} F_{1}\left(\begin{array}{c|c}
-n,-x & \frac{1}{p} \\
-N & (n \geq 0)
\end{array}\right.
$$

is generated by [21]

$$
G(x, t)=(1-p t)^{N}\left(\frac{1+q t}{1-p t}\right)^{x}=\sum_{n=0}^{\infty} \frac{\widetilde{K}_{n}^{p}(x, N)}{n !} t^{n}
$$


where $q$ satisfies $p+q=1$. For this case, we have

$$
A(t)=(1-p t)^{N}, C(t)=\ln \frac{1+q t}{1-p t}, C^{-1}(t)=\frac{e^{t}-1}{e^{t} p-p+1} \quad \text { and } \quad \Phi(t)=\frac{t}{2 p t-1-t} .
$$

It follows that

$$
\mathcal{F}(t)=\frac{A(t)}{A(\Phi(t))} \Phi^{m}(t)=\left(\frac{1-p t}{1-(p-1) t}\right)^{N} \frac{(-t)^{m}}{(1-(2 p-1) t)^{m-N}}
$$

Again, by sum manipulation, we get

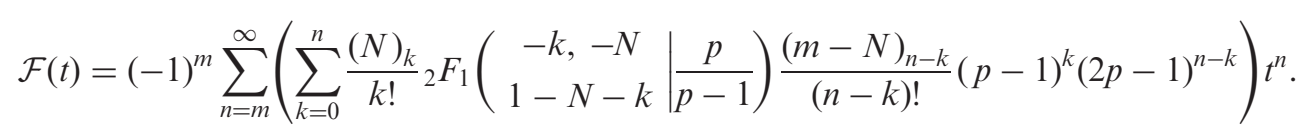

Then the duplication coefficient in (3.16) is given by

$$
C_{m}(n)=(-1)^{m} \frac{n !}{m !}(2 p-1)^{n-m} \sum_{k=0}^{n-m} \frac{(N)_{k}(m-N)_{n-m-k}}{k !(n-m-k) !}\left(\frac{p-1}{2 p-1}\right)^{k}{ }_{2} F_{1}\left(\begin{array}{c}
-k,-N \\
1-N-k
\end{array} \mid \frac{p}{p-1}\right) .
$$

One can find such a representation also by the formula

$$
\widetilde{K}_{n}^{p}(x, N)=\widetilde{M}_{n}\left(x ;-N, \frac{p}{p-1}\right)
$$

which follows from the hypergeometric representations. Therefore from representation (3.21) it follows for the multiplication coefficients of the Krawtchouk polynomials

$$
C_{m}(n)=(-1)^{n} \frac{n !}{m !}(2 p-1)^{n-m} \sum_{k=0}^{n-m} \frac{(-N)_{k}(m-N)_{n-m-k}}{k !(n-m-k) !}\left(\frac{p}{2 p-1}\right)^{k}{ }_{2} F_{1}\left(\begin{array}{c}
-k, N \\
1+N-k
\end{array} \mid \frac{p-1}{p}\right) .
$$

Note that this representation differs from (3.22) modulo some hypergeometric identity.

Using the multsum package, we get the recurrence

$$
\begin{aligned}
& p(m+3)(2 p-1)(p-1)(m+2)(m+1)(m+2-N) C_{m+3}(n)-(m+2)(m+1) \\
& \quad\left(m+1+5 p^{2} m+6 p^{2}-5 p m-6 p-4 p^{2} N+2 N p-n p^{2}+p n\right) C_{m+2}(n) \\
& \quad+(m+1)(4 p m+2 p-2 m-1-2 N p-2 p n+n) C_{m+1}(n) \\
& \quad+(n-m) C_{m}(n)=0
\end{aligned}
$$


w.r.t $m$ with initial values $C_{n}(n)=(-1)^{n}$ and $C_{n+1}(n)=C_{n+2}(n)=0$, as well as

$$
\begin{aligned}
& p(n+2)(n+1)(n+3)(2 p-1)(p-1)(n-N) C_{m}(n)+(n+3)(n+2) \\
& \quad\left(p^{2} m-p m+5 p+5 p n-5 p^{2}-5 n p^{2}-1-n+4 p^{2} N-2 N p\right) \\
& \quad C_{m}(n+1)+(m-n-3) C_{m}(n+3) \\
& -(n+3)(2 p m-m-8 p-4 p n+4+2 n+2 N p) C_{m}(n+2)=0
\end{aligned}
$$

w.r.t $n$.

\section{References}

[1] Area, I., Godoy, E., Ronveaux, A. and Zarzo, A., 2003, Classical discrete orthogonal polynomials, Lah numbers and involutory matrices. Applied Mathematics Letters, 16, 383-387.

[2] Ben Cheikh, Y. and Chaggara, H., 2006, Connection coefficients between Boas-Buck polynomial sets. Journal of Mathematical Analysis and Applications, 319, 665-689.

[3] Ben Cheikh, Y. and Chaggara, H., 2005, Connection coefficients via lowering operators. Journal of Computational and Applied Mathematics, 178, 45-61.

[4] Ben Cheikh, Y. and Chaggara, H., 2006, Linearization coefficients for Sheffer polynomial sets via lowering operators. International Journal of Applied Mathematics and Mathematical Science (In press).

[5] Brafman, F., 1951, Generating functions of Jacobi and related polynomials. Proceedings of the American Mathematical Society, 2, 942-949.

[6] Carlitz, L., 1962, Some multiplication formulas. Rendiconti del Seminario Matematico, 32, $239-242$.

[7] Chaggara, H., Quasi Monomialty and Linearization Coefficients for Sheffer Polynomial Sets (World Scientific, in press).

[8] Chaunday, T.X., 1943, An extension of hypergeometric functions (I). Quarterly Journal of Mathematics, 14, 55-78.

[9] Chihara, T.S., 1978, An Introduction to Orthogonal Polynomials (New York, London, Paris: Gordon and Breach).

[10] Fields, L.J. and Wimp, J., 1961, Expansion of hypergeometric functions in hypergeometric functions. Mathematics of Computation, 15, 390-395.

[11] Gould, W.H. and Hopper, A.T., 1962, Operational formulas connected with two generalizations of Hermite polynomials. Duke Mathematical Journal, 29, 51-63.

[12] Gruntz, D. and Koepf, W., 1995, Maple package on formal power series. Maple Technical Newsletter, 2(2), 22-28

[13] Koekoek, R. and Swarttouw, R.F., 1998, The Askey-Scheme of Hypergeometric Orthogonal Polynomials and its q-Analogue, Technical Report 98-17, Faculty of the Technical Mathematics and Informatics, Delft University of Technology, Delft.

[14] Koepf, W., 1992, Power series in computer algebra. Journal of Symbolic Computation, 13, 581-603.

[15] Koepf, W., 1998, Hypergeometric Summation (Braunschweig-Wiesbaden: Vieweg).

[16] Koepf, W. and Schmersau, D., 1998, Representations of orthogonal polynomials. Journal of Computational and Applied Mathematics, 90, 57-94.

[17] Lewanowicz, S., 1998, The Hypergeometric Function Approach to the Connection Problem for the Classical Orthogonal Polynomials. Technical Report, Institute of Computer Science, University of Wroclaw.

[18] Rainville, E.D., 1960, Special Functions (New York: The Macmillan Company).

[19] Sprenger, T., 2004, Algorithmen für mehrfache Summen, Diploma thesis at the University of Kassel, $1-85$.

[20] Srivastava, H.M. and Manocha, H.L., 1984, A Treatise on Generating Functions, (New York, Chichester, Brisbane, Toronto: John Wiley and Sons).

[21] Szegö, G., 1975, Orthogonal Polynomials, Vol. 23, 4th Edn, American Mathematical Society Colloquium (New York: American Mathematical Society).

[22] Wegschaider, K., 1997, Computer generated proofs of binomial multi-sum identities. Diploma thesis at the J Kepler University of Linz, 1-99. 